\title{
Highly sensitive visual detection of nucleic acid based on a universal strand exchange amplification coupled with lateral flow assay strip
}

\author{
Xiaokun Wang ${ }^{\mathrm{a}}$, Xiudan Wang ${ }^{\mathrm{a}}$, Chao Shi ${ }^{\mathrm{c}}$, Cuiping Ma ${ }^{\mathrm{a}, * *}$, Lingxin Chen ${ }^{\mathrm{b}, \mathrm{d}, *}$ \\ ${ }^{a}$ Shandong Provincial Key Laboratory of Biochemical Engineering, College of Marine Science and Biological Engineering, Qingdao University of Science and Technology, \\ Qingdao, 266042, PR China \\ ${ }^{\mathrm{b}}$ CAS Key Laboratory of Coastal Environmental Processes and Ecological Remediation, Shandong Key Laboratory of Coastal Environmental Processes, Yantai Institute of \\ Coastal Zone Research, Chinese Academy of Sciences, Yantai, 264003, PR China \\ ${ }^{\mathrm{c}}$ College of Life Sciences, Qingdao University, Qingdao, 266071, PR China \\ ${ }^{\mathrm{d}}$ Center for Ocean Mega-Science, Chinese Academy of Sciences, Qingdao, 266071, PR China
}

\section{A R T I C L E I N F O}

\section{Keywords:}

Lateral flow biosensor

Isothermal amplification

Low resource setting

Nucleic acid testing

\begin{abstract}
A B S T R A C T
Amplification reactions coupled with electrophoresis or real-time fluorescence system have been successfully used for the analysis of nucleic acid. However, complex procedures or expensive instruments greatly limit their application in low-resource settings. To address these shortcomings, we fabricated a universal and simple detection platform by integrating strand exchange amplification (SEA) with lateral flow assay (LFA) strip. SEA is a simple isothermal amplification reaction, only requires a pair of primers and one DNA polymerase, above all, its short amplicons are easy to migrate on the strip. LFA strip was proved to be stable for months without large signal deviations and result could be easy to read by naked eyes, which makes it an appropriate option for fieldbased analysis. Our proposed SEA-LFA strip could reliably detect as few as $0.05 \mathrm{nM}$ pork DNA and $0.07 \mathrm{nM}$ duck DNA by the naked eye, its analytical performance is comparable with laboratory-based real-time fluorescence test. Furthermore, this proof-of-concept method could be applied to detect a wide variety of nucleic acid by focusing on primer design rather than on the development of a wholly new analysis platform. We believe that this simple visualization system has great potential as a preliminary test tool in resource-limited areas.
\end{abstract}

\section{Introduction}

Nucleic acid testing (NAT) has received considerable attention due to its critical role in biological studies, medical diagnostics, etc [1,2]. However, the species-specific genes are in low expression abundance genes, nucleic acid amplification methods are required to achieve good detection performance. To date, traditional polymerase chain reaction (PCR) and a variety of isothermal nucleic acid amplification techniques have been successfully applied to the nucleic acid amplification process [3-6]. These techniques have integrating with different kinds of assay approaches to advance the development of sensitive and selective analysis of nucleic acid, such as electrophoresis, real-time fluorescence $[7,8]$. However, these approaches usually based on expensive instruments with laboratory conditions, which largely limit their applications in resource-limited settings, thus, more simple and user-friendly detection platforms are still needed.
Lateral flow assay (LFA) strip, also called dry-reagent strip biosensor, has been widely used for point-of-care (POC) analysis due to its significant advantages, including no sample pre-treatments, user-friendly, short assay time, less interference due to chromatographic separation, relatively low costs, and no requirements for skilled technicians $[9,10]$. Until now, LFA strip has been effectively used to detect proteins [11], toxins [12], nucleic acids [13], etc. In recent years, various nucleic acid amplification methods have coupling with LFA strips for simple nucleic acid analysis with low costs [14-21]. For example, Ellington's group relieved the need of fluorescent readers by integrating loop mediated isothermal amplification (LAMP) to a ubiquitous and low-cost household pregnancy LFA strip, for visual detection of nucleic acid [22]. Cui's group reported the rapid and accurate detection of single nucleotide polymorphisms from genomic DNA samples based on the PCR-LFA platform [23]. PCR is the first and remains the most reliable method for amplifying low abundance nucleic acid to quantities which can be detected [24,25]. However, it requires complex

\footnotetext{
* Corresponding author. CAS Key Laboratory of Coastal Environmental Processes and Ecological Remediation, Shandong Key Laboratory of Coastal Environmental Processes, Yantai Institute of Coastal Zone Research, Chinese Academy of Sciences, Yantai, 264003, PR China.

${ }^{* *}$ Corresponding author. Shandong Provincial Key Laboratory of Biochemical Engineering, College of Marine Science and Biological Engineering, Qingdao University of Science and Technology, Qingdao, 266042, PR China

E-mail addresses: mcp169@163.com (C. Ma), lxchen@yic.ac.cn (L. Chen).
} 
thermal cycling steps to mediate denaturation, annealing and extension [26-28]. In contrast, LAMP reaction can be carried out at a constant temperature and shows very high sensitivity and selectivity [28-30], but it requires multiple primers. Besides, the PCR and LAMP amplicons are usually more than a hundred bases pair [31], it's too large to migrate on the LFA strip, signal transduction processes were needed [22], which leading to high cost and complex design procedures. Therefore, despite the advances in technology, more economical and easy-to-operate nucleic acid amplification methods are still needed, especially in low-resource areas.

Strand exchange amplification (SEA) is a simple isothermal amplification method [32], it takes advantage of the DNA duplex's dynamically dissociate at appropriate reaction temperature, leading the local opening of base pairs to create a single-stranded denaturation bubble [33-35]. Until now, SEA has been successfully used to detect pBlu2KSP dsDNA, Escherichia coli 16S rRNA and meat adulteration based on the real-time fluorescence detection instrument or fluorescence dye colorimetric assay $[32,36]$. Compared with other isothermal amplification techniques, SEA has more advantages, for example, it's only requires one pair of primers and a DNA polymerase, more important, short amplicons (less than $50 \mathrm{bp}$ ) are more suitable to migrate on the LFA strip. Herein, we developed a universal and simple on-site nucleic acid detection method based on SEALFA strip, which relieved the need of expensive instrument and special reaction environment. In this work, pork DNA was used to assess the feasibility, the biotin labelled forward primer and digoxin labelled backward primer invade to the single-stranded denaturation bubble and extend with the DNA polymerase to generate the dsDNA SEA amplicons. Subsequently, the generated SEA amplicons were transfer to the LFA strip and induced the anti-digoxin-gold nanoparticles (AuNPs) accumulate in concentration on the test line until they were visually detectable. Such a detection approach does not require any sample preparation or complex instruments, only needs a portable heating block (i.e. small metal or water bath) and relies on visual detection, avoids the inherent ambiguity of fluorescence outputs. To verify the universality, we also successfully detected duck genomic DNA using the same LFA strip. All assays were independently validated using the real-time PCR assay. To the best of our knowledge, this is the first report of universal and visual detection of nucleic acid based on SEA-LFA platform. This proposed method holds great potential for on-site analysis in the areas of food security, disease diagnostic and environment monitoring, especially in the low resource underserved communities.

\section{Experimental section}

\subsection{Materials and reagents}

Pork, duck, mutton, chicken and beef were purchased from local supermarket (Qingdao, China). Tissue genomic DNA extraction kits were purchased from Tiangen Biotech Co., Ltd (Beijing, China). SEA kit was purchased from Navid Biotechnology Co., Ltd. (Qingdao, China). Molecular weight marker and loading buffer were purchased from Takara (Dalian, China). Bst 2.0 WarmStartTM DNA polymerase was purchased from New England Biolabs. Eva Green was purchased from Bridgen (Beijing, China). The nitrocellulose (NC) membrane attached to a backing card was purchased from Millipore Corporation (Billerica. MA, USA). The ultrapure water $\left(18 \mathrm{M} \Omega \mathrm{cm}^{-1}\right)$ used in this work was obtained from a Milli-Q water purification system (Millipore Corporation, MA, USA). Sequences were synthesized by Sangon Biotech (Shanghai, China) and were listed in Table S1. Other reagents were purchased from Sigma-Aldrich (St. Louis, MO, USA). All reagents were of analytical reagent grade and were used without further purification.

\subsection{Instrumentation}

The electrophoresis data was obtained with a ChampGel5000 imaging system (Sage Creation Science Co, LTD, Beijing, China). The realtime fluorescence result was acquired using a CFX96 ${ }^{\mathrm{TM}}$ Real-Time detection system (Bio-Rad, CA, USA). UV-Vis absorption spectra were obtained with Cary 100 spectrophotometer (Varian, Salt Lake City, UT, USA). Dynamic light scattering (DLS) data was obtained with a NanoZS90 apparatus (Malvern Instruments, Malvern, UK). High-magnification transmission electron microscopy (TEM) image was acquired using a JEOL JEM 2100F instrument (JEOL, Tokyo, Japan). Scanning electron microscopy (SEM) images were obtained with a MIRA3 instrument (TESCAN, Brno, Czech Republic). All strips were prepared using a programmable cutter (HGS201, Autokun, China).

\subsection{Preparation of anti-digoxin antibody conjugated gold nanoparticles (AuNPs)}

AuNPs were prepared according to the previously reported kinetically controlled seed growth method [37]. Thermometer and condenser were used to control the temperature and prevent the evaporation of the mixtures, respectively. Au seeds were synthesized by heating $75 \mathrm{~mL}$ of $2.2 \mathrm{mM}$ sodium citrate in a three-necked round-bottomed flask with rapid magnetic stirring. As soon as boiling, $0.5 \mathrm{~mL}$ of $25 \mathrm{mM} \mathrm{HAuCl}_{4}$ was added. The mixture changed from yellow to bluish grey and then to soft pink color in $10 \mathrm{~min}$. The resulting Au seeds were cooled to $90{ }^{\circ} \mathrm{C}$ and then $0.5 \mathrm{~mL}$ of $60 \mathrm{mM}$ sodium citrate and $0.5 \mathrm{~mL}$ of $25 \mathrm{mM} \mathrm{HAuCl}_{4}$ were sequentially added every $2 \mathrm{~min}$. This process was repeated 12 times and the resultant solution was kept stirring under $90{ }^{\circ} \mathrm{C}$ for $30 \mathrm{~min}$.

Antibody-conjugated AuNPs were prepared according to our previous work with slight modifications [38]. Briefly, $5 \mu \mathrm{L}$ of $0.1 \mathrm{mM}$ DHLA was added to $1 \mathrm{~mL}$ AuNPs colloid, the mixture was incubated at room temperature for $2 \mathrm{~h}$. Unreacted chemical reagents were removed by centrifugation at $5000 \mathrm{rpm}$ for $10 \mathrm{~min}$ and dispersed in $10 \mathrm{mM}$ PBS buffer solution. Subsequently, $5 \mu \mathrm{L}$ of 1-ethyl-3-(3-(dimethylamino)propyl) carodiimide (EDC)/N-hydroxysuccinimide (NHS) was added and incubated $40 \mathrm{~min}$ at room temperature, excess EDC/NHS was separated by centrifugation, and then $4 \mu \mathrm{L}$ anti-digoxin antibody was reacted with the mixture for $2 \mathrm{~h}$. Finally, nonspecific binding antibodies were removed by centrifugation at $5000 \mathrm{rpm}$ for $10 \mathrm{~min}$, and the remaining colloid was suspended in PBS buffer solution containing 5\% BSA, $0.25 \%$ Tween 20 , and $10 \%$ sucrose.

\subsection{Preparation of the probe immobilized LFA strip}

The LFA strip was assembled according to our previous study with slight modifications $[39,40]$. The sample pad was blocked with $0.25 \%$ TRITON ${ }^{\circledR} \mathrm{X}-100$ in $2 \mathrm{mM}$ PBS followed by overnight drying at $37{ }^{\circ} \mathrm{C}$ to ensure optimal conditions for the analytes throughout the flux. The test line and control line on the $\mathrm{NC}$ membrane were prepared by dispensing $2 \mathrm{mg} / \mathrm{mL}$ of $\mathrm{SA}$ and $0.15 \mathrm{mg} / \mathrm{mL}$ of anti-mouse $\mathrm{IgG}$, respectively. $10 \times$ anti-digoxin-AuNPs were added on the conjugate pad. The membrane and conjugate pad were dried at room temperature. All the components were assembled on the plastic backing layer, each component overlapped $2 \mathrm{~mm}$ to ensure solution migration, and then cut into $3.8 \mathrm{~mm}$-wide strips.

\subsection{SEA-LFA procedure}

Double-stranded DNA (dsDNA) is very stable due to the complementarity of many base pairs, the key point of PCR technique is repeatedly increasing and lowering the reaction temperature to mediate denaturation, annealing and extension of the initiate dsDNA [3]. In fact, the interaction of each base pair of dsDNA is relatively weak to allow intermittent breaking of base pairs to create a single stranded denaturation bubble at a suitable temperature, even at room or physiological temperature $[34,41,42]$. The forming rate of bubbles is heavily dependent on the GC content and the temperature. SEA is like the traditional PCR technique, employs one pair of primers and a DNA polymerase to initiate the exponential amplification, moreover, the entire SEA reaction was performed at a constant temperature by taking 
advantage of the DNA duplex's "breathing" formed unwound singlestranded denaturation bubble. During this process, the forward primer anneals to the bubble of dsDNA and extends by the Bst 2.0 WarmStartTM DNA polymerase. Due to the spontaneous local conformational fluctuations within the amplicons, the backward primer invades the bubble of the amplicons and extends to provide the dsDNA amplicons of these two primers. Subsequently, the generated dsDNA amplicons and primers continue to produce new generations of dsDNA amplicons $[32,36]$. SEA was carried out in a $15 \mu \mathrm{L}$ reaction system, without pretreatment. The mixture containing SEA kit reagents and different concentration of target DNA, $1.0 \times 10^{-6} \mathrm{M}$ biotin-P1 and digoxin-P2 was incubated at $62{ }^{\circ} \mathrm{C}$ for $50 \mathrm{~min}$. Subsequently, $1 \mu \mathrm{L}$ of diluted SEA amplicons mixed with $49 \mu \mathrm{L}$ of running buffer, the LFA strips were dipped into the mixture solution and pictures were taken after $15-20 \mathrm{~min}$ by using a cell phone (iPhone 8X, Apple, CA, USA).

\section{Results and discussion}

\subsection{Principle of the SEA-LFA strip for detection of nucleic acid}

The operating principle of SEA-LFA strip for nucleic acid detection was illustrated in Fig. 1. It consisted of two steps: (1) isothermal SEA reaction; (2) detect SEA amplicons by LFA strip. In this work, pork DNA was chosen as the target to verify the feasibility. We designed biotin labelled forward primer (biotin-P1) and digoxin labelled backward primer (digoxin-P2) targeting the pork DNA. As shown in Fig. 1a, in the presence of pork DNA, biotin-P1 and digoxin-P2 invaded to the singlestranded denaturation bubble at the suitable temperature, and extend with the DNA polymerase to generate numerous biotin and digoxin labelled dsDNA SEA amplicons with the length of 42 bp (Fig. 1b(i)). For the LFA strip, as shown in Fig. 1b, it consisted of four sections: a sample pad, a conjugate pad, an absorption pad and a NC membrane with plastic backing card. Streptavidin and anti-mouse IgG were immobilized on the test line and control line of the NC membrane through a strong electrostatic interaction [40,43], respectively. Anti-digoxin antibody was immobilized on the surface of AuNPs through a chemical bond and stored in the conjugate pad.

(a)

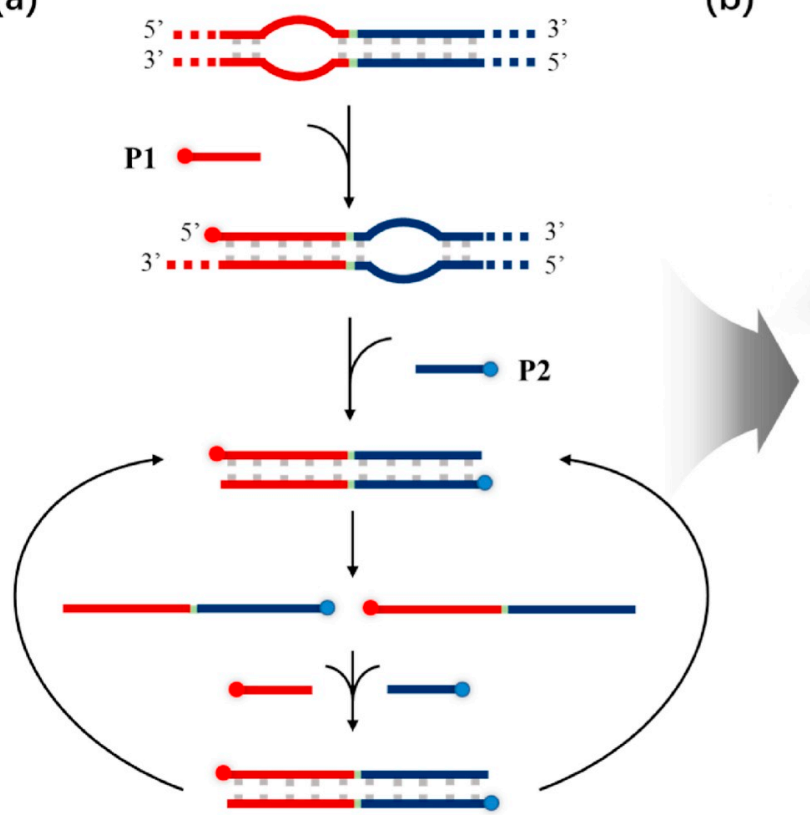

After the loading of SEA amplicons on the sample pad, the solution migrated toward the absorption pad and conjugate pad by the capillary force, the biotin and digoxin labelled dsDNA would specifically bind to anti-digoxin-AuNPs forming numerous conjugates (Fig. 1b (ii)). When these conjugates passed through the test line, they would be captured by the streptavidin that were pre-immobilized on the test line (Fig. 1b (iii)), the sandwich structure formed on the test line would produce an obvious visible signal. The excess conjugates continued to migrate on the NC membrane, and captured by the control line through anti-mouse IgG reacted with anti-digoxin-antibody (Fig. $1 \mathrm{~b}$ (iv)). In contrast, if pork DNA was not present in the sample and no specific SEA amplification reaction taken place, then no sandwich structure occurred on the test line, only the control line showed red color. In brief, in the presence of pork DNA, two red lines were observed ("on") as a positive result. In the absence of pork DNA, only one red line was observed on the control line ("off") as a negative result, the red color on the control line was used to demonstrate the SEA-LFA strip is working as intended.

\subsection{Characterization of anti-digoxin antibody conjugated AuNPs}

Fig. 2 shows the sequential process for fabricating anti-digoxinAuNPs. DHLA was used as a linker for the conjugation of anti-digoxin antibody. Briefly, two-SH groups of DHLA were cleaved and bonded to the surface of AuNPs and the - $\mathrm{COOH}$ terminal groups of DHLA were activated by EDC and NHS. Subsequently, anti-digoxin antibody was immobilized on the surface of AuNPs [38]. The morphology of AuNPs was characterized by transmission electron microscopy (TEM), diameters of the AuNPs in the TEM image were analyzed using Image $\mathrm{J}$ software created at the National Institutes of Health (U.S.), the average particle diameter was estimated to be $38.6 \mathrm{~nm}$. UV-Vis spectra of the pure AuNPs was shown in Fig. S1. The diameter was determined directly from UV-Vis spectra using Haiss's equation [44]:

$d=\exp \left(3 \frac{A_{s p r}}{A_{450}}-2.2\right)$

Using this equation, the diameter was estimated to be $44.7 \mathrm{~nm}$. Based on these results, the diameter of AuNPs was estimated to be

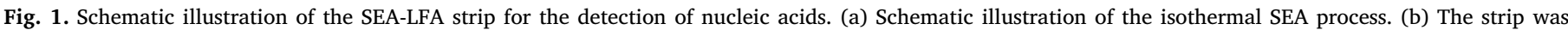

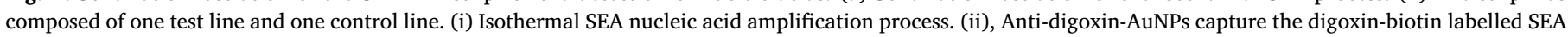

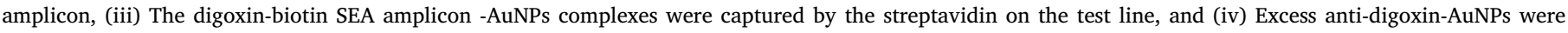
captured by the anti-mouse IgG on the control line. 
(a)

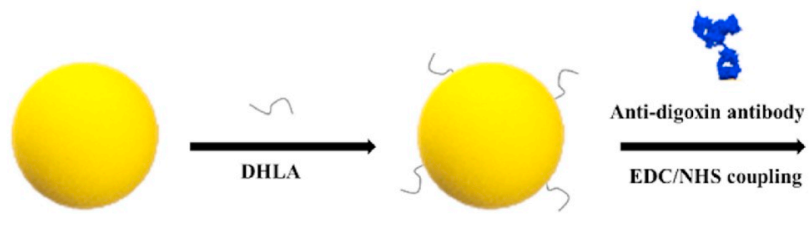

(b)

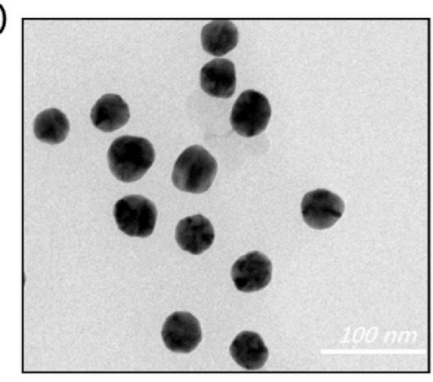

(c)

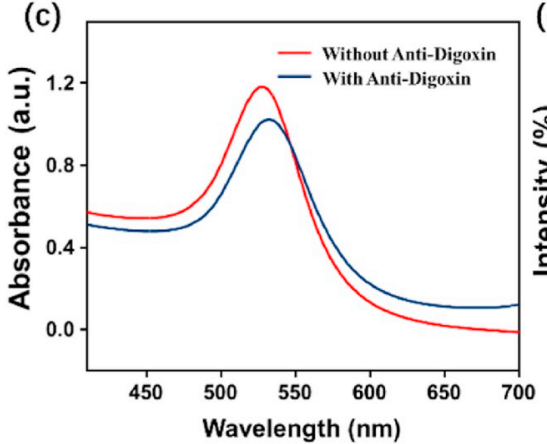

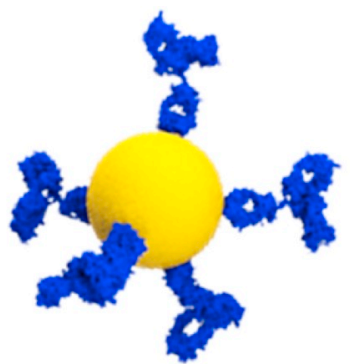

(d)

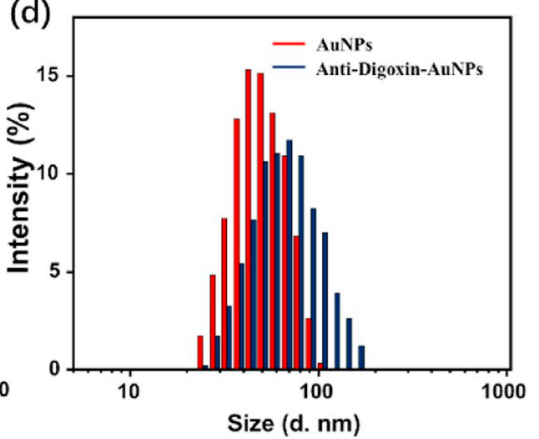

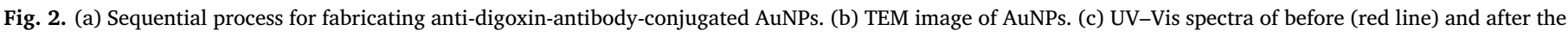

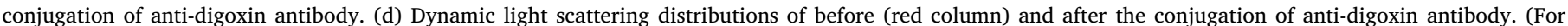
interpretation of the references to color in this figure legend, the reader is referred to the Web version of this article.)

$40 \pm 5 \mathrm{~nm}$. In addition, the effects of antibody on the surface of the AuNPs were estimated by UV-Vis spectrophotometry and dynamic light scattering (DLS) measurements. As shown in Fig. 2c and d, the surface plasmon band shifting from $527 \mathrm{~nm}$ to $531 \mathrm{~nm}$, the average hydrodynamic size measured by DLS also increased after antibody conjugation. These spectral changes in UV-Vis spectrophotometry and DLS confirmed that antibodies were successfully immobilized on the surface of AuNPs.

\subsection{Specificity of the SEA-LFA strip}

Different kinds of nucleic acid samples, including pork, beef, mutton, chicken, duck and blank control were processed through the SEA-LFA system, to evaluate the specificity. The SEA process was carried out with a simple metal bath under the same conditions, after incubation, the generated amplicons were transferred to the LFA strip, respectively. The result digital photographic images and relative intensity of all the six samples were displayed in Fig. 3a and b. Refer to strip 1 and strip 6, an "on-off" qualitative result could be obvious distinguished by the naked eye. SEM images of the "on-off" conditions of test lines on the LFA strip were shown in Fig. 4, which demonstrated the accumulation of anti-digoxin-AuNPs induced a characteristic red band. Strip 2 to strip 5 showed the results of samples with beef, mutton, chicken and duck nucleic acids, respectively. There was no red color on the test lines, and the red control lines confirmed that the strip was working. In addition, laboratory-based real-time fluorescence test and PAGE electrophoresis were used to double check the SEA process, as shown in Fig. 3c and d, only target could initiate the SEA reaction and generate the dsDNA SEA amplicons. All these results demonstrate that the SEA reaction and the SEA-LFA detection platform show excellent specificity toward the target.

\subsection{Optimization of the SEA-LFA platform parameters}

To achieve an optimal detection condition for the SEA-LFA platform, SEA reaction temperature and running buffer were systematically investigated. Optimization process was carried out by changing one parameter while keeping others constant.
The forming rate of single-stranded denaturation bubbles is heavily dependent on the GC content and the temperature [35]. According to the $\mathrm{Tm}$ values of the designed primers, real-time fluorescence curves of SEA processes were measured at $60{ }^{\circ} \mathrm{C}, 61{ }^{\circ} \mathrm{C}, 62{ }^{\circ} \mathrm{C}, 63{ }^{\circ} \mathrm{C}$ and $64{ }^{\circ} \mathrm{C}$ with same concentration of pork DNA, respectively. As shown in Fig. $\mathrm{S} 2$, there was no false positive at different reaction temperature and the amplification efficiency at $62{ }^{\circ} \mathrm{C}$ was much better than other reaction temperature. So, $62{ }^{\circ} \mathrm{C}$ was chosen as the optimum amplification temperature used in the following experiment.

Running buffer strongly affects the migration of anti-digoxin-AuNPs and the appropriate buffer solution could improve the binding efficiency of anti-digoxin antibody, digoxin-biotin SEA amplicons and streptavidin. As shown in Fig. S3, we investigated the performance of the SEA-LFA strip with three different types of buffer solutions: Tris$\mathrm{HCl}, \mathrm{PBS}$, and $2 \times$ SSC. In addition, $1 \%$ BSA was added to minimize the edge effect. The relative intensity represented the peak area ratio of test line to control line of each strip. According to the result, it could be seen that the on/off ratio value by using $2 \times$ SSC buffer solution containing $1 \%$ BSA was significantly higher than the other two buffers. Therefore, $2 \times$ SSC buffer solution containing $1 \%$ BSA was determined to be the final buffer solution for the following experiments.

\subsection{Analytical performance of the SEA-LFA}

The analytical capability of the SEA-LFA strip was investigated with six different concentration of pork DNA ranging from blank to $50 \mathrm{nM}$ by using the strategy described in Fig. 1. The response of SEA-LFA strips to the SEA amplicons obtained from different concentration of pork DNA was shown in Fig. 5a. Under the optimized experiment conditions, we could detect as few as $0.05 \mathrm{nM}$ pork DNA by naked eyes. Fig. $5 \mathrm{~b}$ shows the corresponding quantitation results of strips by using Image J software, the relative intensity represented the peak area ratio of test line and control line, error bars indicated the standard deviations from three measurements. Moreover, to evaluate the detection capability of the SEA-LFA strip, the laboratory-based real-time fluorescence test was also performed for different concentration of pork DNA. Real-time fluorescence curves were displayed in Fig. 5c. According to the experimental result, we could find that the proposed SEA-LFA strip and real-time 
(a)

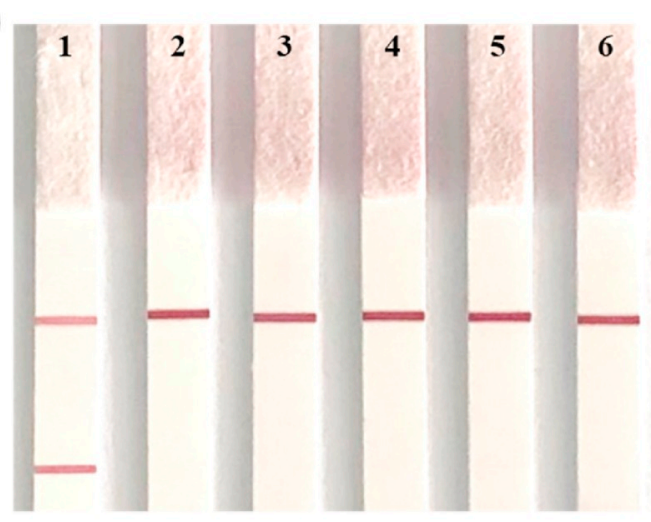

(c)

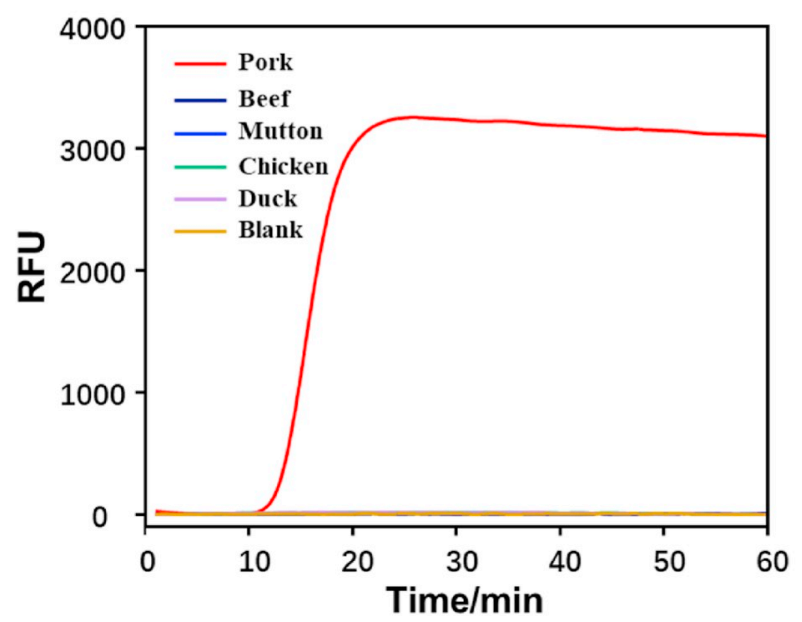

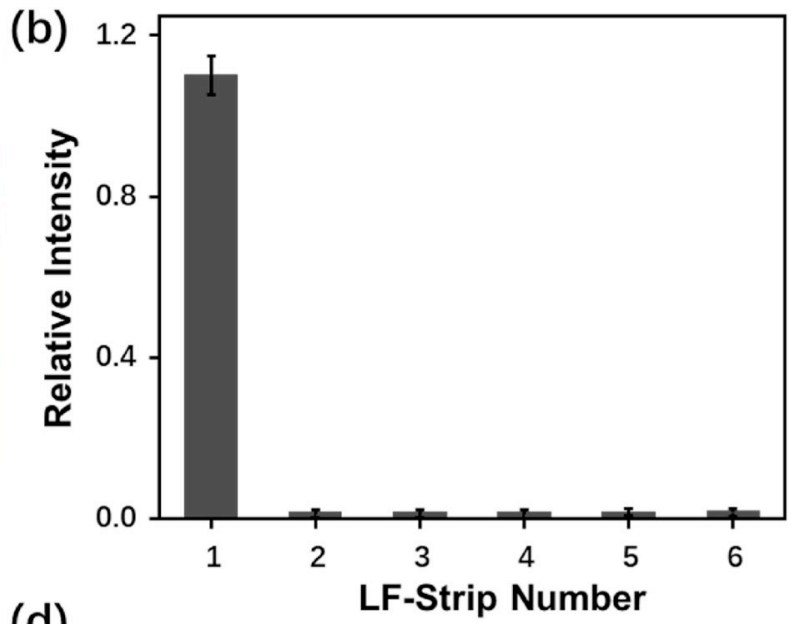

(d)

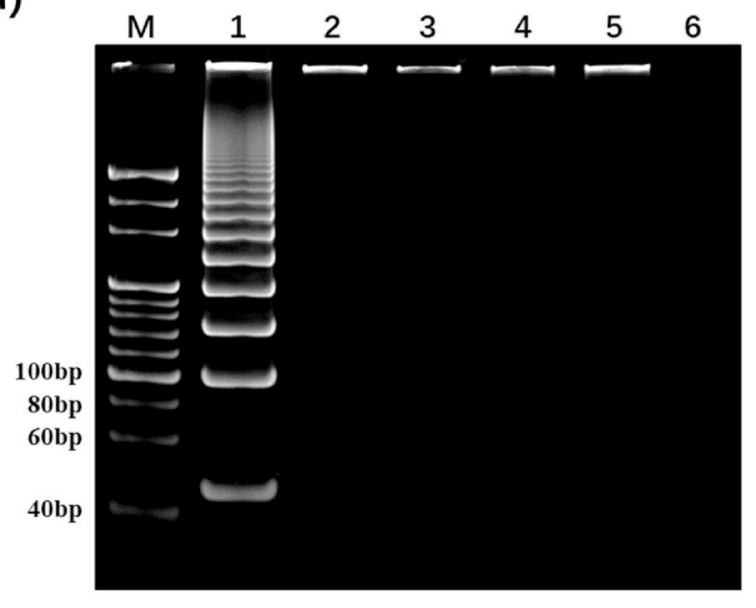

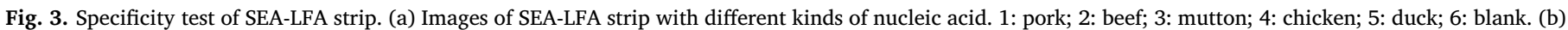

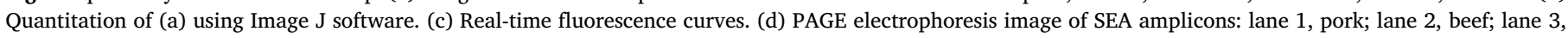
mutton; lane 4, chicken; lane 5, duck; lane 6, blank control.

fluorescence test demonstrate comparable detection performance.

Reproducibility is of great importance in practical applications. The reproducibility of the SEA-LFA strip was investigated by measuring the same concentration of pork DNA with three replicates. As shown in Fig. S4, similar responses could be obtained, the intra-day relative standard deviations (RSD) value was calculated to be $2.27 \%$. To further evaluate the accuracy of the proposed SEA-LFA strip, a receiver operating characteristic (ROC) curve (Fig. S5) was calculated based on 20 positive and 10 negative samples. The ROC curve of SEA-LFA strip showed an area under the curve (AUC) of 0.973 with a sensitivity of $95 \%$. These results demonstrated that SEA-LFA strip showed satisfactory accuracy and intra-day repeatability.

Next, the detection performance of SEA-LFA strip in mixture samples was investigated, four binary mixtures containing $0 \%, 5 \%, 10 \%$ and $20 \%$ $(\mathrm{w} / \mathrm{w})$ of pork in beef were prepared to a final weight of $200 \mathrm{~g}$ and mashed, respectively. The mixture DNA was extracted by the tissue genomic DNA extraction kit, Fig. S6 and Fig. 6 show the laboratory-based real-time fluorescence result and the response of SEA-LFA strips to the SEA (a)

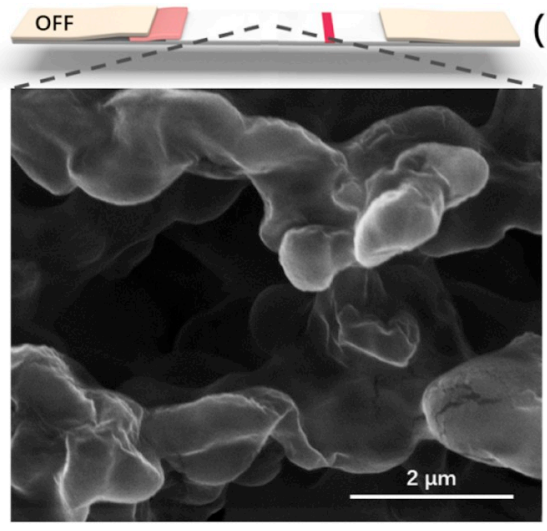

(b)

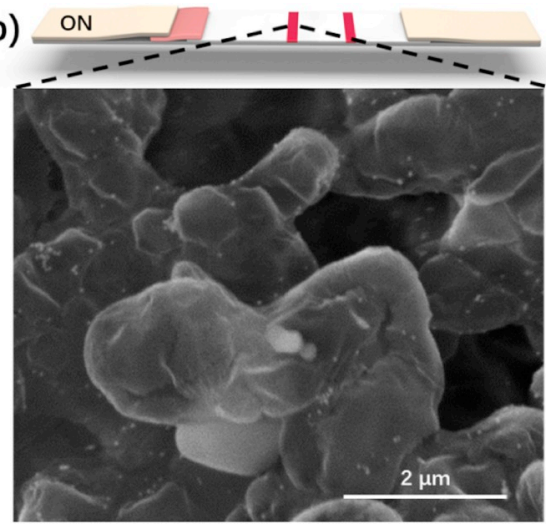

Fig. 4. SEM images of the SEA-LFA strips in the absence ("off") and presence ("on”) of pork nucleic acid. 
(a)

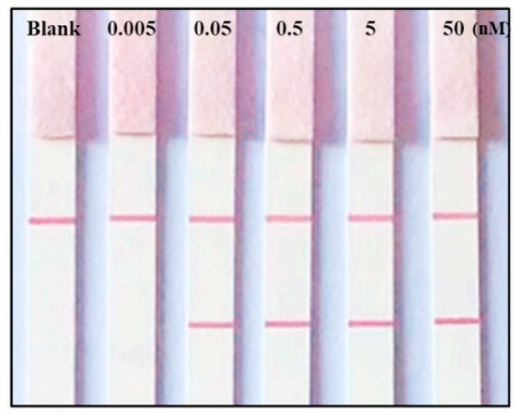

(b)

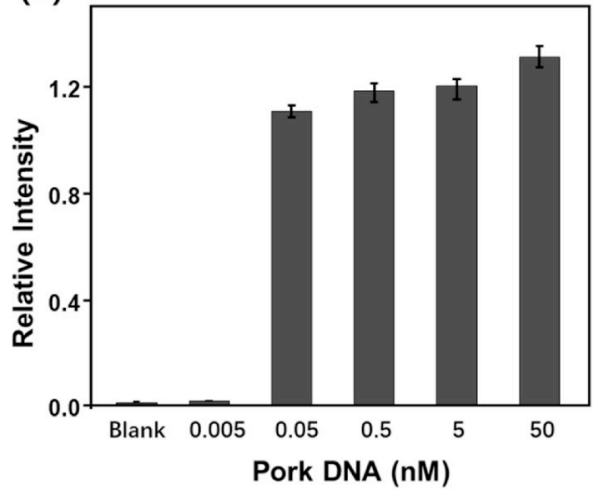

(c)

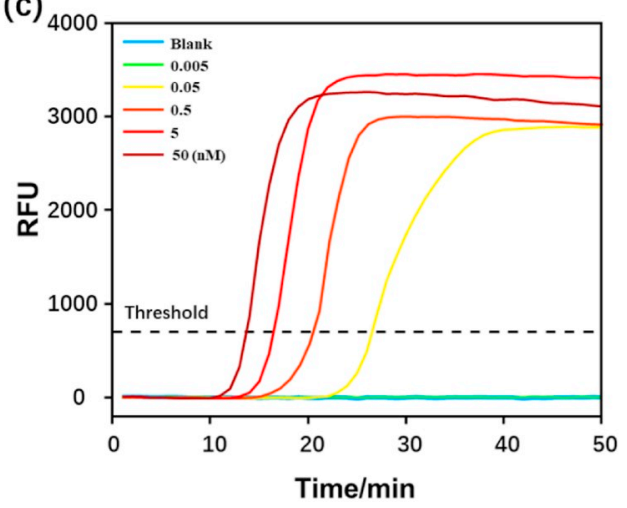

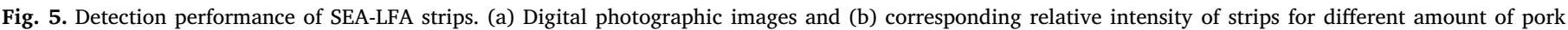

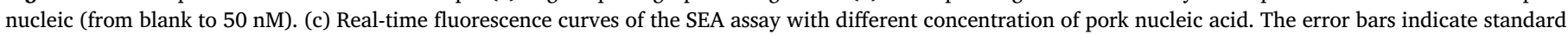
deviations of three measurements.

(a)

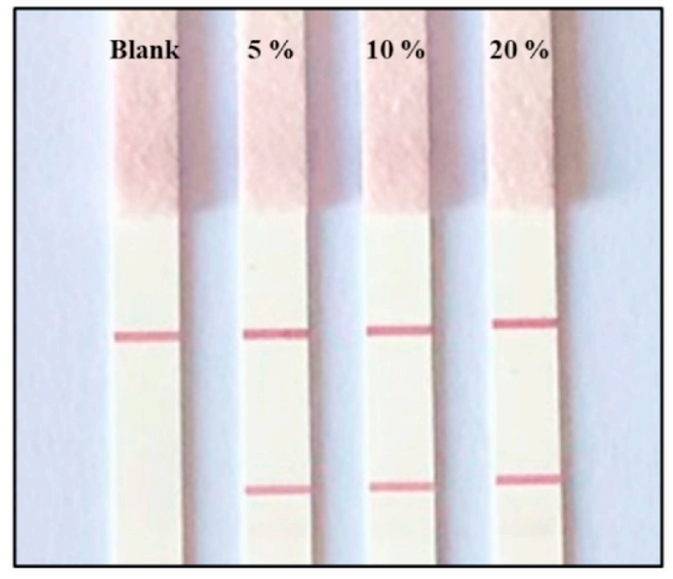

(b)

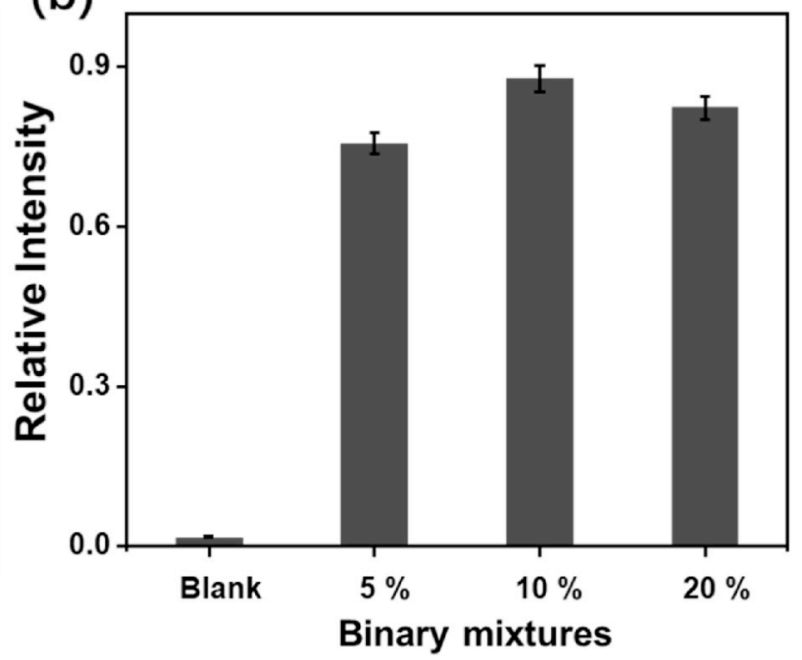

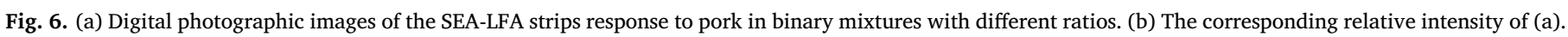
The error bars indicated standard deviations of three measurements.

amplicons obtained from the extracted mixture DNA, respectively. According to images, we could find that $5 \%$ mixture samples could produce an obvious, easy to read positive signal on the test line. These results demonstrated that proposed SEA-LFA strip could reliably distinguish pork from mixture samples and get comparable detection selectivity with the instrument-based method. Additionally, as shown in Fig. S7, the proposed SEA-LFA strip could successfully detect as few as $0.07 \mathrm{nM}$ duck DNA, which demonstrate this proof-of-concept method could be applied to detect a wide variety of nucleic acid by focusing on primer design rather than on the development of a wholly new analysis platform. In addition, the stability of SEA-LFA strip was also checked. According to Fig. S8, the signal intensity was down $6.2 \%$ after 30 days and $11.5 \%$ after 60 days, the inter-day repeatability was calculated to be $6.12 \%$. This universal and simple strip was stored for at least 60 days without large signal deviations.

\section{Conclusions}

In summary, we developed a universal and robust SEA-LFA strip for the detection of nucleic acid by the naked eye. It is composed of a simple isothermal SEA reaction and a user-friendly LFA strip. The presence of target DNA could produce numerous biotin and digoxin labelled SEA amplicons, subsequently identified through a simple color change in the test line of the LFA strip. The SEA amplicons have short bases pair (less than $50 \mathrm{bp}$ ) are easy to migrate on the strip, more importantly, this proof-of-concept method could be applied to detect a wide variety of nucleic acid by focusing on primer design rather than on the development of a wholly new analysis platform. According to the experimental result, this proposed method could reliably detect as few as $0.05 \mathrm{nM}$ pork DNA and $0.07 \mathrm{nM}$ duck DNA using the naked eye. The AUC, intra and inter-day repeatability demonstrate that SEA-LFA strip showed satisfactory accuracy and stability.

Compared with the PCR integrated LFA, this proposed SEA-LFA strip does not require the sequential thermo-cycling processes, only need a portable heating block (Table S2). In addition, SEA process requires only a pair of primers and one Bst DNA polymerase. Compared with other isothermal amplification integrated LFAs, SEA-LFA relieves the need of various accessory proteins (polymerase) or multiple primers (4 or 6) to perform strand separation and amplification (Table S2), which reduce the cost and simplify the primer design process. These features greatly simplify the SEA-LFA implementation in point-of-care analysis devices. We believe that this simple visualization system will provide new insights into the detection of nucleic acids without access to infrastructure or laboratories.

\section{CRediT authorship contribution statement}

Xiaokun Wang: Conceptualization, Methodology, Writing - original draft, Writing - review \& editing. Xiudan Wang: Data curation, 
Investigation. Chao Shi: Validation. Cuiping Ma: Supervision, Resources, Writing - review \& editing. Lingxin Chen: Supervision, Project administration.

\section{Declaration of competing interest}

The authors declare no competing financial interest.

\section{Acknowledgments}

This work was financially supported by grants from the National Natural Science Foundation of China (21904078, 31670868, 21976209), Key Deployment Project of Centre for Ocean MegaResearch of Science, Chinese Academy of Science (COMS2019J01) and Taishan Scholar Project Special Funding (No.ts20190962).

\section{Appendix A. Supplementary data}

Supplementary data to this article can be found online at https:// doi.org/10.1016/j.talanta.2020.120978.

\section{References}

[1] H. Peng, A.M. Newbigging, Z. Wang, J. Tao, W. Deng, X.C. Le, H. Zhang, DNAzymemediated assays for amplified detection of nucleic acids and proteins, Anal. Chem. 90 (2017) 190-207.

[2] A. Niemz, T.M. Ferguson, D.S. Boyle, Point-of-care nucleic acid testing for infectious diseases, Trends Biotechnol. 29 (2011) 240-250.

[3] R.K. Saiki, S. Scharf, F. Faloona, K.B. Mullis, G.T. Horn, H.A. Erlich, et al., Enzymatic amplification of beta-globin genomic sequences and restriction site analysis for diagnosis of sickle cell anemia, Science 230 (1985) 1350-1354.

[4] T. Notomi, H. Okayama, H. Masubuchi, T. Yonekawa, K. Watanabe, N. Amino, et al., Loop-mediated isothermal amplification of DNA, Nucleic Acids Res. 28 (2000) e63-e.

[5] J.C. Guatelli, K.M. Whitfield, D.Y. Kwoh, K.J. Barringer, D.D. Richman, T.R. Gingeras, Isothermal, in vitro amplification of nucleic acids by a multienzyme reaction modeled after retroviral replication, P. Natl. Acad. Sci. USA 87 (1990) 1874-1878.

[6] Y. Zhao, F. Chen, Q. Li, L. Wang, C. Fan, Isothermal amplification of nucleic acids, Chem. Rev. 115 (2015) 12491-12545.

[7] P. Craw, W. Balachandran, Isothermal nucleic acid amplification technologies for point-of-care diagnostics: a critical review, Lab Chip 12 (2012) 2469-2486.

[8] U. Reischl, H.-J. Linde, M. Metz, B. Leppmeier, N. Lehn, Rapid identification of methicillin-resistantStaphylococcus aureus and simultaneous species confirmation using real-time fluorescence PCR, J. Clin. Microbiol. 38 (2000) 2429-2433.

[9] P. Brangel, A. Sobarzo, C. Parolo, B.S. Miller, P.D. Howes, S. Gelkop, et al., A serological point-of-care test for the detection of IgG antibodies against Ebola virus in human survivors, ACS Nano 12 (2018) 63-73.

[10] D. Quesada-González, A. Merkoçi, Nanoparticle-based lateral flow biosensors, Biosens. Bioelectron. 73 (2015) 47-63.

[11] A. Zamora-Gálvez, E. Morales-Narváez, J. Romero, A. Merkoçi, Photoluminescent lateral flow based on non-radiative energy transfer for protein detection in human serum, Biosens. Bioelectron. 100 (2018) 208-213.

[12] S. Song, N. Liu, Z. Zhao, E. Njumbe Ediage, S. Wu, C. Sun, et al., Multiplex lateral flow immunoassay for mycotoxin determination, Anal. Chem. 86 (2014) 4995-5001.

[13] X. Fu, Z. Cheng, J. Yu, P. Choo, L. Chen, J. Choo, A SERS-based lateral flow assay biosensor for highly sensitive detection of HIV-1 DNA, Biosens. Bioelectron. 78 (2016) 530-537.

[14] J. Reboud, G. Xu, A. Garrett, M. Adriko, Z. Yang, E.M. Tukahebwa, et al., Paperbased microfluidics for DNA diagnostics of malaria in low resource underserved rural communities, P. Natl. Acad. Sci. USA 116 (2019) 4834-8342.

[15] Ö. Akinedena, T. Wittwer, K. Geister, M. Plötz, E. Usleber, Nucleic acid lateral flow immunoassay (NALFIA) with integrated DNA probe degradation for the rapid detection of Cronobacter sakazakii and Cronobacter malonaticus in powdered infant formula, Food Contr. 109 (2020) 106952

[16] Z. Wang, T. Li, W. Yu, L. Qiao, S. Yang, A. Chen, A low-cost novel lateral flow nucleic acid assay (LFNAA) for yak milk authentication, Lebensm. Wiss. Technol. 122 (2020) 109038

[17] Y.M. Zhang, Y. Zhang, K.B. Xie, Evaluation of CRISPR/Cas12a-based DNA detection for fast pathogen diagnosis and GMO test in rice, Mol. Breed. 40 (2020) 1-12.

[18] I.V. Safenkova, A.V. Ivanov, E.S. Slutskaya, A.V. Samokhvalov, A.V. Zherdev, B.B. Dzantiev, Key significance of DNA-target size in lateral flow assay coupled with recombinase polymerase amplification, Anal. Chim. Acta 1102 (2020) 109-118.

[19] A.V. Ivanov, I.V. Safenkova, A.V. Zherdev, B.B. Dzantiev, Nucleic acid lateral flow assay with recombinase polymerase amplification: solutions for highly sensitive detection of RNA virus, Talanta 210 (2020) 120616.

[20] H. Jayan, H.B. Pu, D.W. Sun, Recent development in rapid detection techniques for microorganism activities in food matrices using bio-recognition: a review, Trends Food Sci. Technol. 95 (2020) 233-246.

[21] M.Y. Zhang, J. Ye, J.S. He, F. Zhang, J.F. Ping, C. Qian, J. Wu, Visual detection for nucleic acid-based techniques as potential on-site detection methods. A review, Anal. Chim. Acta 1099 (2020) 1-15.

[22] Y. Du, A. Pothukuchy, J.D. Gollihar, A. Nourani, B. Li, A.D. Ellington, Coupling sensitive nucleic acid amplification with commercial pregnancy test strips, Angew. Chem. Int. Ed. 56 (2017) 992-996.

[23] W. Hui, S. Zhang, C. Zhang, Y. Wan, J. Zhu, G. Zhao, et al., A novel lateral flow assay based on GoldMag nanoparticles and its clinical applications for genotyping of MTHFR C677T polymorphisms, Nanoscale 8 (2016) 3579-3587.

[24] P. Donnelly, Progress and challenges in genome-wide association studies in humans, Nature 456 (2008) 728.

[25] S.Q. Tsai, A.J. Iafrate, J.K. Joung, Genome editing: a tool for research and therapy: towards a functional understanding of variants for molecular diagnostics using genome editing, Nat. Med. 20 (2014) 1103.

[26] S. Yang, R.E. Rothman, PCR-based diagnostics for infectious diseases: uses, limitations, and future applications in acute-care settings, Lancet Infect. Dis. 4 (2004) 337-348.

[27] D.A. Thomson, E.H. Tee, N.T. Tran, M.J. Monteiro, M.A. Cooper, Oligonucleotide and polymer functionalized nanoparticles for amplification-free detection of DNA, Biomacromolecules 13 (2012) 1981-1989.

[28] J. Li, J. Macdonald, Advances in isothermal amplification: novel strategies inspired by biological processes, Biosens. Bioelectron. 64 (2015) 196-211.

[29] J. Kampeera, P. Pasakon, C. Karuwan, N. Arunrut, A. Sappat, S. Sirithammajak, et al., Point-of-care rapid detection of Vibrio parahaemolyticus in seafood using loop-mediated isothermal amplification and graphene-based screen-printed electrochemical sensor, Biosens. Bioelectron. 132 (2019) 271-278.

[30] X. Zhang, S.B. Lowe, J.J. Gooding, Brief review of monitoring methods for loopmediated isothermal amplification (LAMP), Biosens. Bioelectron. 61 (2014) 491-499.

[31] V.c. Ruiz-Valdepeñas Montiel, M.L. Gutiérrez, R.M. Torrente-Rodríguez, E. Povedano, E. Vargas, A.J. Reviejo, et al., Disposable amperometric polymerase chain reaction-free biosensor for direct detection of adulteration with horsemeat in raw lysates targeting mitochondrial DNA, Anal. Chem. 89 (2017) 9474-9482.

[32] C. Shi, F. Shang, M. Zhou, P. Zhang, Y. Wang, C. Ma, Triggered isothermal PCR by denaturation bubble-mediated strand exchange amplification, Chem. Commun. 52 (2016) 11551-11554.

[33] J. Fei, T. Ha, Watching DNA breath one molecule at a time, P. Natl. Acad. Sci. USA 110 (2013) 17173-17174.

[34] G. Altan-Bonnet, A. Libchaber, O. Krichevsky, Bubble dynamics in double-stranded DNA, Phys. Rev. Lett. 90 (2003) 138101.

[35] A. Hanke, R. Metzler, Bubble dynamics in DNA, J. Phys. Math. Gen. 36 (2003) L473.

[36] R.X.D. Liu, X.J. Wang, Y. Wang, J. Shi, C. Shi, W. Wang, C.P. Ma, A simple isothermal nucleic acid amplification method for the effective onsite identification for adulteration of pork source in mutton, Food Contr. 98 (2019) 297-302.

[37] N.G. Bastús, J. Comenge, V. Puntes, Kinetically controlled seeded growth synthesis of citrate-stabilized gold nanoparticles of up to $200 \mathrm{~nm}$ : size focusing versus Ostwald ripening, Langmuir 27 (2011) 11098-11105.

[38] X. Wang, S.G. Park, J. Ko, X. Xiao, V. Giannini, S.A. Maier, et al., Sensitive and reproducible immunoassay of multiple mycotoxins using surface-enhanced Raman scattering mapping on 3D plasmonic nanopillar arrays, Small 14 (2018) 1801623.

[39] M.K. Tsang, W. Ye, G. Wang, J. Li, M. Yang, J. Hao, Ultrasensitive detection of ebola virus oligonucleotide based on upconversion nanoprobe/nanoporous membrane system, ACS Nano 10 (2016) 598-605.

[40] J. Hwang, S. Lee, J. Choo, Application of a SERS-based lateral flow immunoassay strip for the rapid and sensitive detection of staphylococcal enterotoxin B, Nanoscale 8 (2016) 11418-11425.

[41] T.S. VanErp, S. Cuesta-Lopez, M. Peyrard, Bubbles and denaturation in DNA, Eur. Phys. J. E: Soft Matter Biol. Phys. 20 (2006) 421-434.

[42] S. Talukder, P. Chaudhury, R. Metzler, S.K.J. Banik, Determining the DNA stability parameters for the breathing dynamics of heterogeneous DNA by stochastic optimization, Chem. Phys. 135 (2011) 1651.

[43] X. Wang, N. Choi, Z. Cheng, J. Ko, L. Chen, J. Choo, Simultaneous detection of dua nucleic acids using a SERS-based lateral flow assay biosensor, Anal. Chem. 89 (2017) 1163-1169.

[44] W. Haiss, N.T.K. Thanh, J. Aveyard, D.G. Fernig, Determination of size and concentration of gold nanoparticles from UV-Vis spectra, Anal. Chem. 79 (2007) 4215-4221. 